\title{
DESCRIÇÃO DA LARVA DE DIASTATOPS OBSCURA (FABRICIUS) (ODONATA, LIBELLULIDAE)
}

\author{
N.D. Santos 1 \\ J.M. Costa ${ }^{2}$ \\ J.R. Pujol-Luz ${ }^{3}$
}

\begin{abstract}
DESCRIPTION OF THE LARVA OF DIASTATOPS OBSCURA (FABRICIUS) (ODONATA, LIBELLULIDAE). The larva of Diastatops obscura (Fabricius, 1775) is described and figured based on exuviae of last instar of reared specimes collected on still waters in São João river, Silva Jardim $\left(22^{\circ} 38^{\prime}-42^{\circ} 18^{\prime}\right)$, Rio de Janeiro, Brazil. The relationship among the larva of $\mathbf{D}$. obscura and larvae of Celithemis are discussed.

KEY WORDS. Odonata, Libellulidae, Diastatops obscura, taxonomy larva
\end{abstract}

O gênero neotropical Diastatops Rambur, 1842, apresenta sete espécies descritas (MONTGOMERY, 1940, DAVIES \& TOBIN, 1985), sendo D. obscura (Fabricius, 1775) a única espécie a possuir algum registro de sua forma imatura.

SANTOS (1980) apresentou uma descrição sucinta da larva de $D$. obscura, fornecendo os caracteres observados nas imagos emergidas em laboratório, além de assimilar as semelhanças e diferenças observadas entre esta larva e as larvas de Perithemis Hagen, 1861; Celithemis Hagen, 1861 (Libellulidae) e Aeschnosoma Selys, 1870 (Cordulidae).

ARANGO \& ROLDAN (1983) figuraram uma larva não identificada (Fig. 10:93, "Libellulidae A") representando em detalhe a larva de último estádio. Os caracteres apresentados nas figuras permitiram relacionar esta larva ao gênero Diastatops, provavelmente $D$. obscura.

Neste artigo, a larva de $D$. obscura é descrita e figurada com base em seis exúvias de último estádio de exemplares criados em laboratório, provenientes do Rio São João, Município de Silva Jardim $\left(22^{\circ} 38^{\prime}-42^{\circ} 18^{\prime}\right)$, Rio de Janeiro, Brasil. As semelhanças citadas por SANTOS (1980) entre as larvas de D. obscura e as de Celithemis são discutidas através dos dados disponíveis na literatura.

1) Obra póstuma.

2) Departamento de Entomologia, Museu Nacional do Rio de Janeiro, Quinta da Boa Vista, 20940-040 Rio de Janeiro, Rio de Janeiro, Brasil.

3) Departamento de Biologia Animal, Universidade Federal Rural do Rio de Janeiro, 23851-970 Itaguaí, Rio de Janeiro, Brasil. 


\section{Diastatops obscura (Fabricius, 1775)}

Figs 1-12

\section{Larva de pequeno porte; coloração uniformemente ocrácea.}

Cabeça larga, com olhos compostos em posição antero-lateral, de forma cônica, ultrapassando o bordo lateral da cabeça; margem occipital arredondada rodeada por cerdas dispostas irregularmente até a margem dos olhos; tubérculos frontais pouco desenvolvidos, formando dois nódulos entre os olhos compostos; antenas com sete segmentos, comprimento relativo dos antenômeros 30-21-27-18-30-39-33; fórmula mandibular $\frac{L}{R} \frac{1234}{1234} \frac{O}{y} \frac{a b}{a b d}$; lábio em forma de cálice em vista ventral com pequenas manchas marron escuras esparsas, quando dobrado ultrapassando a base do segundo par de pernas; pré-mento com 11 setas de cada lado, margem anterior com cinco pares de setas espiniformes, dois pares de setas espiniformes entre a sutura do pré-mento e o palpo labial; palpo labial com manchas marron escuras esparsas; palpo esquerdo com sete setas; palpo direito com oito setas e uma setela; garra móvel pouco desenvolvida; margem externa do palpo com pequenas cerdas; margem interna com as seis digitações próximas a garra móvel profundas e irregulares; palpo esquerdo com a primeira digitação bifứcada; cada digitação apresentando um único espinho terminal; a partir da sexta digitação a margem interna torna-se suavemente crenulada, suportando a intervalos irregulares grupos de espinhos.

Tórax com apófise protorácica supracoxal bifurcada, guarnecida por cerdas pequenas; tecas alares atingindo o nível do sexto segmento abdominal; impressões das nervuras evidentes; pernas longas e finas, conferindo à larva um aspecto araneiforme; fêmures suportando uma longa cerda espiniforme no terço distal; superfície das pernas guarnecidas por pequenas cerdas.

Abdome cilíndrico, sendo o sexto segmento o mais largo; margem lateral externa provida de pequenas cerdas concentradas em toda sua extensão; espinhos dorsais presentes do terceiro ao nono segmentos, sendo os três primeiros curtos em série crescente de tamanho, os sexto ao nono segmentos, longos, curvos e aproximadamente do mesmo tamanho; espinhos laterais presentes nos segmentos oito e nove, sendo os do nono cerca de quatro vezes os do oitavo em comprimento, cercos curtos e ponteagudos; epiprocto com extremidade distal espiniforme, paraproctos de forma triangular, ultapassando a extremidade do epiprocto.

Medidas (em milímetros). Comprimento total: 18,5; comprimento da cabeça: 2,42; largura máxima da cabeça: 4,28; largura máxima do pré-mento: 3,40 ; comprimento do pré-mento: 3,73 ; teca alar anterior: 4,00 ; teca alar posterior: 4,48; fêmures (F1: 3,20; F2: 4,00; F3 4,80); tíbias (T1: 3,68; T2: 4,64: T3: $6,56)$; comprimento total do abdome: 11,0 ; comprimento do espinho do oitavo segmento do abdome: 0,83 ; comprimento do espinho do nono segmento do abdome: 3,32 ; comprimento do epiprocto: 0,81 ; comprimento do paraprocto: 0,84 ; comprimento do cerco: 0,42 . 

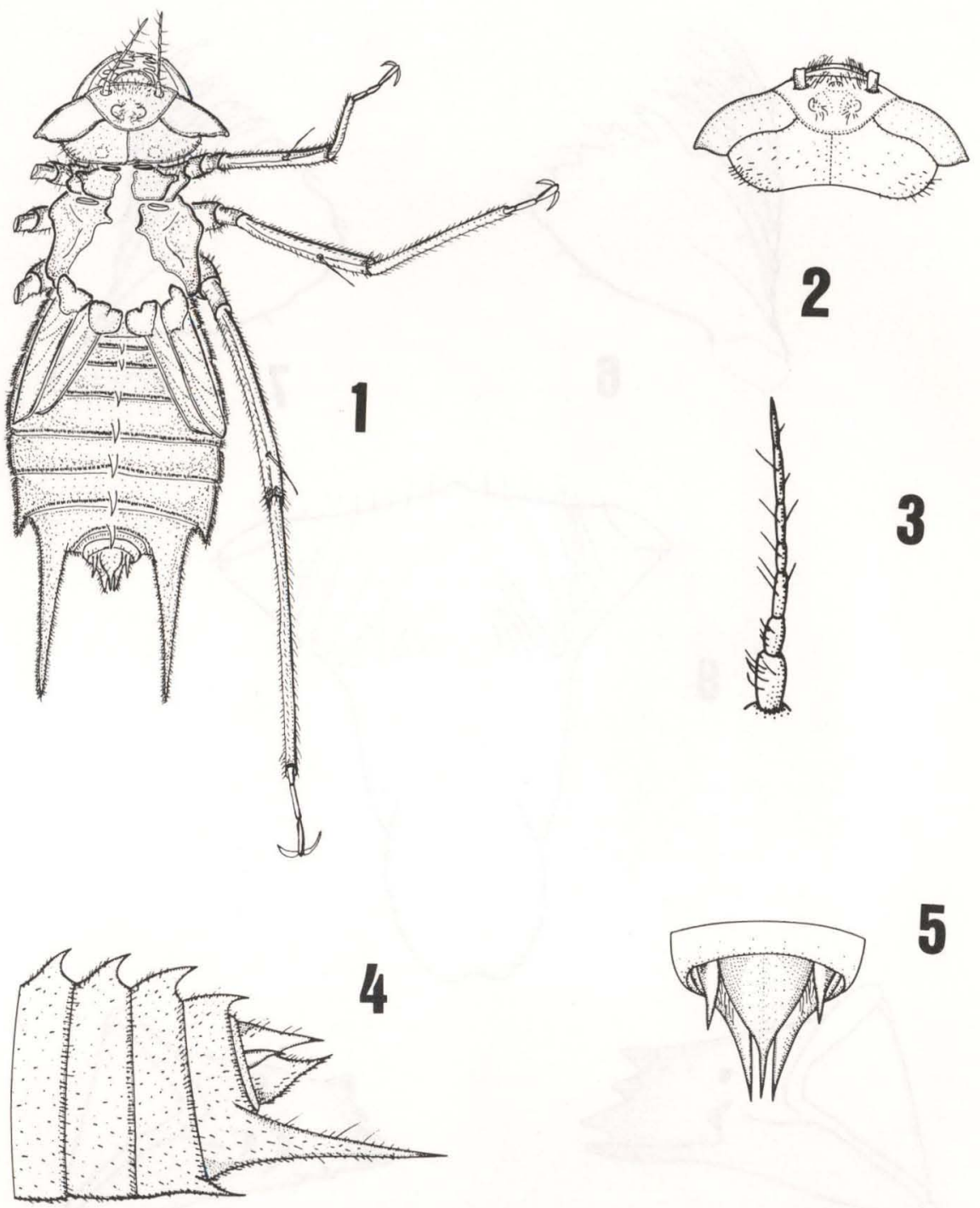

5

Figs 1-5. Diastatops obscura. (1) Vista dorsal da exúvia de último estádio; (2) vista dorsal da cabeça; (3) antena esquerda; (4) vista lateral do abdome; (5) vista dorsal da pirâmide caudal.

Material examinado. BRASIL, Rio de Janeiro, Silva Jardim, Rio São João, N.D. Santos leg.; 5-II-1977, $\mathrm{n}^{\circ}$ 384, um macho (emergência 10-II-1977); 5-III1977, $\mathrm{n}^{\circ}$ 376, um macho (emergência 14-III-1977) e $\mathrm{n}^{\circ}$ 380, uma fêmea (emergência 8-III-1977); 16-IX-1978, no 1249, um macho e no 1271, um macho (emergência 24-X-1978); 14-X-1978, no 378, uma fêmea (emergência 16-XII-1978); material depositado na coleção do Museu Nacional, Universidade Federal do Rio de Janeiro. 

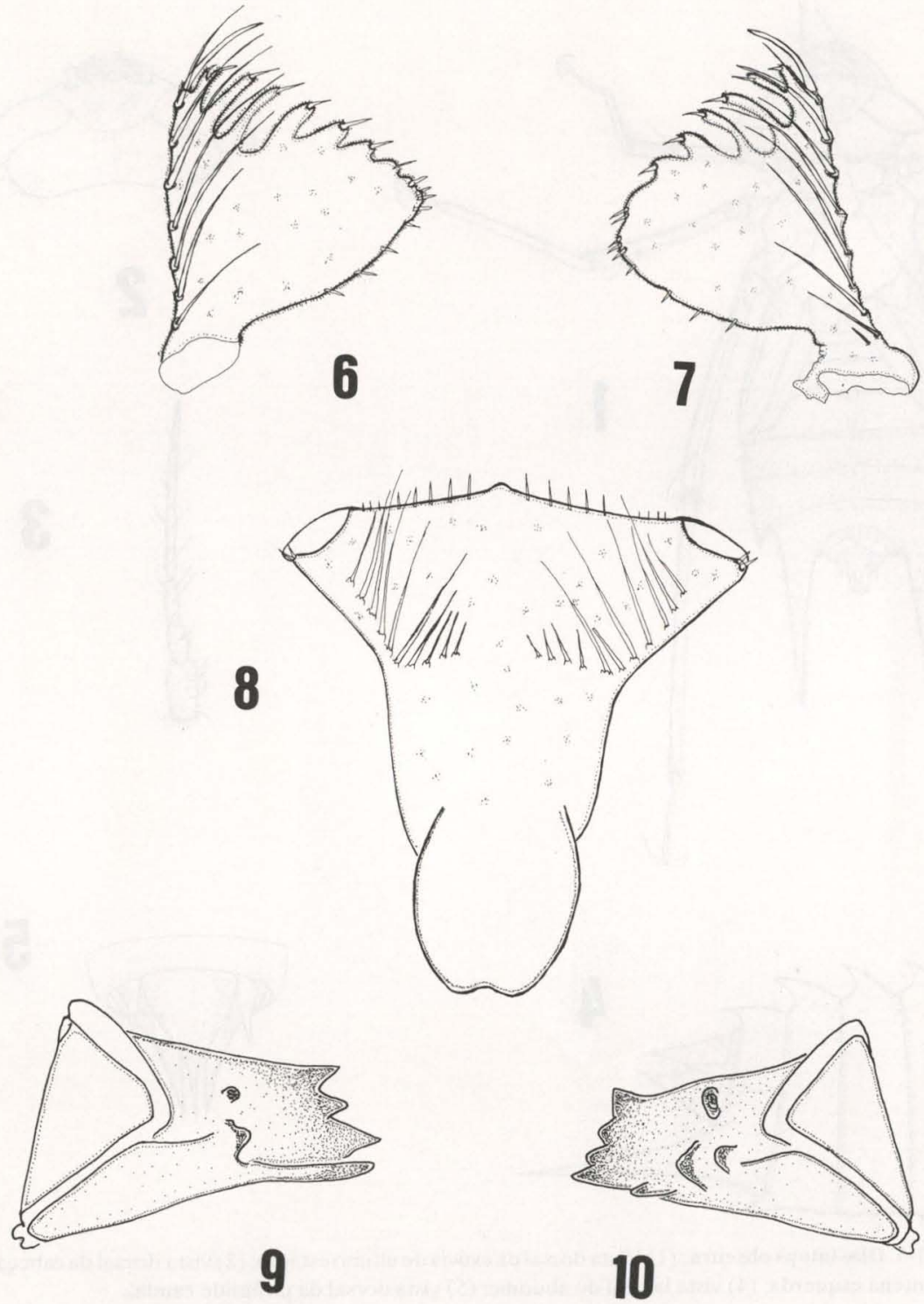

Figs 6-10. Diastatops abscura. (6) Vista dorsal do palpo labial esquerdo; (7) vista dorsal do palpo labial direito; (8) vista dorsal do pré-mento; (9) superfície interna da mandibula esquerda; (10) superfície interna da mandibula direita.

\section{DISCUSSÃO}

A larva de D. obscura apresenta características pouco comuns entre os libelulídeos, como: olhos compostos de forma cônica; tubérculos frontais presentes; palpos labiais com profundas digitações assimétricas. 
Entre os libelulídeos, a presença de olhos compostos de forma cônica, projetados além da margem lateral da cabeça, só foi observada em quatro espécies de Celithemis, gênero que compreende nove espécies de distribuição neártica (DAVIES \& TOBIN, 1985).

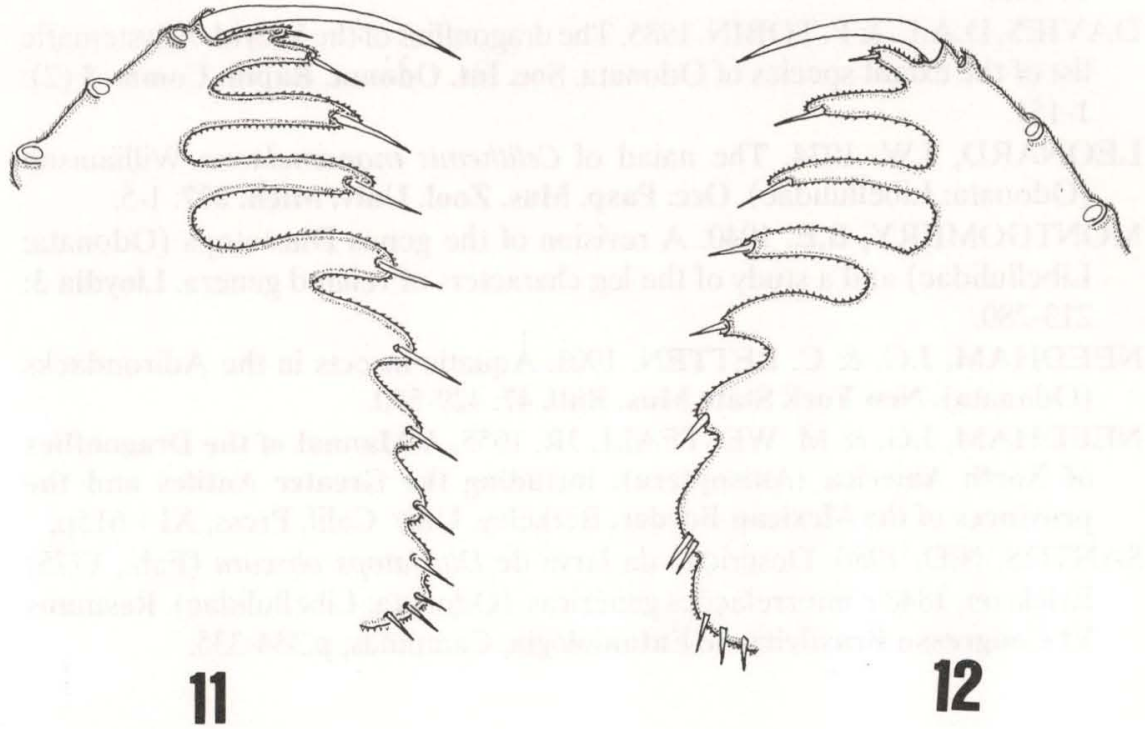

Figs 11-12. Diastatops abscura. (11) Margem interna do palpo labial esque rdo; (12) margem interna do palpo labial direito.

NEEDHAM \& WESTFALL (1955) utilizaram o caráter "forma dos olhos compostos" na primeira dicotomia de uma chave para larvas das espécies de Celithemis, representando dois grupos: o primeiro com olhos cônicos - "C. fasciata Kirby, 1889; C. monomelaena Williamson, 1910; C. ornata (Rambur, 1842) e $C$. verna Pritchard, 1935"; o segundo com olhos arredondados - "C. eponina (Drury, 1773); C. amanda (Hagen, 1861); C. martha ? Williamson, 1922; C. bertha Williamson, 1922 e C. elisa (Hagen, 1861)".

Uma comparação entre os caracteres das larvas de $D$. obscura e os dois grupos de Celithemis, considerados neste trabalho, foi realizada com base na literatura disponível (NEEDHAM \& BETTEN, 1901; LEONARD, 1934; NEEDHAM \& WESTFALL, 1955). Exceto pela forma cônica dos olhos compostos nas larvas do primeiro grupo de espécies de Celithemis, os três caracteres acima descritos formam uma combinação exclusiva de $D$. obscura, considerando-se que nas larvas de Celithemis estes caracteres refletem os estados mais comuns observados entre os Libellulidae - olhos compostos arredondados, ausência de tubérculos frontais e palpo labial crenulado. Uma diferença marcante entre as larvas destes dois gêneros seria o número de espinhos dorsais no abdome: quarto ao sétimo em Celithemis e terceiro ao nono segmentos em $D$. obscura. 


\section{REFERÊNCIAS BIBLIOGRÁFICAS}

ARANGO, M.C. \& G.P. ROLDAN. 1983. Odonatos inmaturos del Departamento de Antioquia en diferentes pisos altitudinales. Actual. Biol. 12 (46): 91-105.

DAVIES, D.A.L. \& P. TOBIN. 1985. The dragonflies of the World: A systematic list of the extant species of Odonata. Soc. Int. Odonat. Rapid. Comm. 5 (2): 1-151.

LEONARD, J.W. 1934. The naiad of Celithemis monomelaena Williamson (Odonata: Libellulidae). Occ. Pasp. Mus. Zool. Univ. Mich. 297: 1-5.

MONTGOMERY, B.E. 1940. A revision of the genus Diastatops (Odonata: Libellulidae) and a study of the leg characters of related genera. Lloydia 3: 213-280.

NEEDHAM, J.G. \& C. BETTEN. 1901. Aquatic insects in the Adirondacks (Odanata). New York State Mus. Bull. 47: 429-540.

NEEDHAM, J.G. \& M. WESTFALL JR. 1955. A Manual of the Dragonflies of North America (Anisoptera), including the Greater Antiles and the provinces of the Mexican Border. Berkeley, Univ. Calif. Press, XI +615p.

SANTOS, N.D. 1980. Descrição da larva de Diastatops obscura (Fab., 1775) Erickson, 1848 e interrelações genéricas. (Odonata: Libellulidae). Resumos VI Congresso Brasileiro de Entomologia, Campinas, p.334-335.

Recebido em 30.12.1991; aceito em 25.XI.1993. 